\title{
A Method Analyzing Aerosol Particle Shape and Scattering Based on Imaging
}

\author{
Shiyong Shao, Yinbo Huang and Ruizhong Rao
}

Additional information is available at the end of the chapter

http://dx.doi.org/10.5772/48314

\section{Introduction}

Aerosol particle shape is a key parameter affecting its physical characters, especially scattering properties ${ }^{[1]}$. The information of shape reveals important application in such fields as atmospheric radiation and remote sensing, climate research, radar meteorology ${ }^{[2]}$. The convenient availability and simplicity of the Lorenz-Mie theory has resulted in a widespread practice of treating non-spherical particles as if they were spheres to which Lorenz-Mie results are applicable. However, the assumption of sphere is rarely made after first having studied the effects of non-sphere and concluded that they are negligible but is usually based on a perceived lack of practical alternatives ${ }^{[3]}$.

In a variety of occupational, environmental and industrial scenarios, particles within the size range from a few tenths of a micrometer to a few hundred micrometers play an important role $^{[4]}$. Since the majority of aerosol particles are to some extent non-spherical and indicating relation with their origins, the knowledge of particles' shape may be used to judge the source of those particles and hence facilitate more effective contamination control and to reduce inadvertent particle generation. For example, fibrous particles are often corresponding to textile industry, flake-like particles corresponding to papermaking industry, etc.

The scattering profile of light scattered by any particle is determined by its size parameter, its shape, and its orientation with respect to the incident illumination ${ }^{[4]}$. The spatial intensity distribution of scattered light thus contains information by which the particle may often be classified or even identified. The light scattering suits to be used for deducing shape of aerosol particles by detecting scattering information, which is rapid and non-contact ${ }^{[5-7]}$. By analyzing pairs of signal from opposite detectors, Diehl differentiate bluffly the shape of suspending particles ${ }^{[8]}$. Bartholdi reflected majority of scattering light onto a circular photodiodes array, and gained more abundant information about particle shape ${ }^{[9]}$. Kaye 
assessed the feasibility of classifying individual aerosol particles on the basis of size and shape parameters, which determined by measurement and analysis of the spatial intensity distribution of laser radiation scattered by the particle shown in Fig. $1^{[10,11]}$.

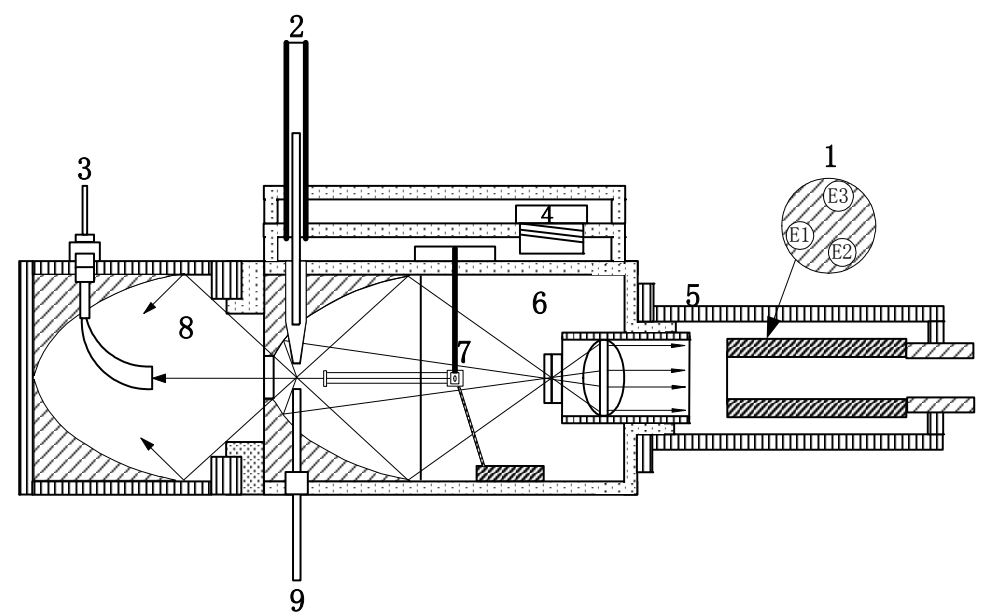

1.Detector Channels E1,E2,E3 2.Sample Inlet 3.Detector Channel E4 4.Filter for Sheath Air 5.Photomultiplier House 6.Main-Chamber 7.Laser and Modulating System 8.Rear-Chamber 9.Pump

Figure 1. Schematic diagram of the aerosol shape analyzer

The laser beam is directed onto the particle flow by a small $45^{\circ}$ mirror supported by an optical window. Particle-laden air is drawn in through the scattering chamber in laminar flow and is ensheathed by filtered air drawn in through ports simultaneously ${ }^{[12]}$. Individual particles in the sample air transverse the laser beam and produce pulses of scattered light. Three miniature photomultipliers are incorporated an arrangement to allow measurement of variations in azimuthal scattering from individual airborne particles between $28^{\circ}$ and 141 to the beam incident direction upon an ellipsoidal reflector whose primary focus is coincident with the scattering volume. The output of detector E4 measures the forwards scattering used in estimation of the particle size. The first developments to achieve this are incorporated in Biral's ASAS ${ }^{\mathrm{TM}}$ technology and has been developed by the UK armed forces. The instrument allows airborne particles in the sub-10 $\mu \mathrm{m}$ size range to be classified into size and shape classes in real-time at rates of up to about 10000 particles per second.

Although the instruments described above are able to differentiate between spherical and non-spherical particles, also provide some crude indication of particle shape, the full potential of spatial intensity scattering analysis for non-spherical particle characterization should only be realized by the detailed analysis both of azimuthal and of polar scattered intensity variations.

To observe micro-particle, the microscopy is often preferred instrument, which represents an excellent technique for directly examining target ${ }^{[13]}$. However, for manual microscopy, 
elaborate sample preparation is necessary and only a few particles can be examined resulting in very low statistical relevance of the data. Recently, a faster evaluation of activated sludge floc properties became possible by connecting the microscope to automated image analysis software ${ }^{[14]}$, see Fig.2.

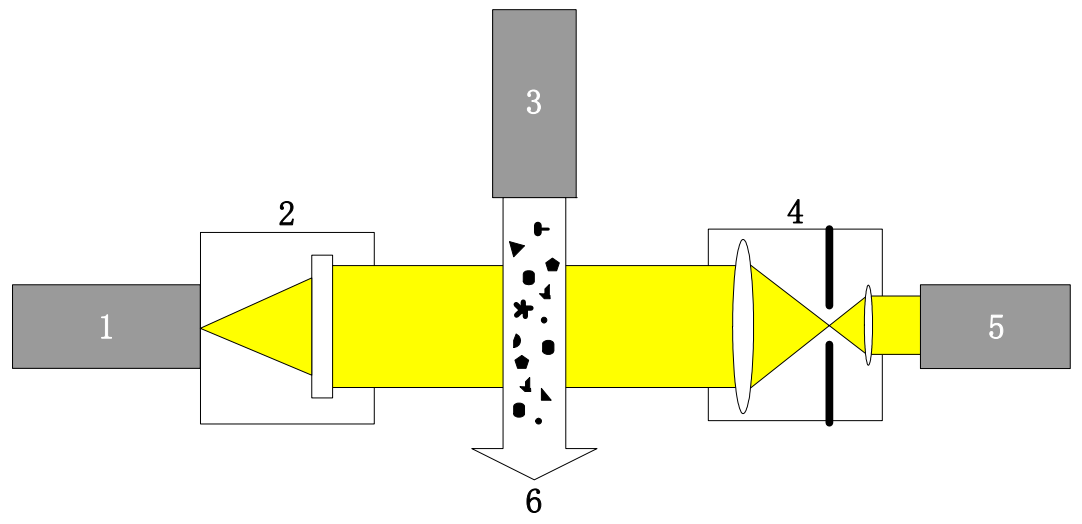

1. Light Source 2.Light Modulation 3.Sample Dispersion 4.Imaging Object Lens 5.CCD with microscope 6.Particle Stream

Figure 2. Sketch map of the microscope CCD system

The light of a pulsed light source is expanded by a beam expansion unit, which creates a parallel beam. The dispersed particle flow is illuminated and finally imaged by an CCD via microscope. The particles show arbitrary orientation and the number of overlapping particles are lost. The light source creates stable visible light pulses about $1 \mathrm{~ns}$ at power of about $0.15 \mathrm{~nJ} /$ pulse. The repetition rate is adjustable from 1 to $500 \mathrm{~Hz}$ meeting the specifications of the high speed CMOS camera. One image is composed out of $1024 \times 1024$ pixels of $10 \mu \mathrm{m} \times 10 \mu \mathrm{m}$ area with 256 gray levels. Imaging objectives for different magnifications are mounted on a carousel for simple selection of a measuring range by software.

Light scattering and imaging by CCD via microscope are routine two methods for detecting aerosol particle shape. CCD video microscope is volume-based, and light scattering is number -based ${ }^{[15-17]}$. If a number distribution fits a log-normal distribution, then its transformation to volume distribution will result in another log-normal distribution, characterized by ${ }^{[18]}$ :

$$
\ln x_{g} \bullet V=\ln x_{g} \bullet N+3 \ln ^{2} \sigma_{g}
$$

$x_{g}$ is the geometric mean of the distribution, $\sigma_{g}$ is the geometric standard deviation, $V$ is volume based distribution and $N$ is number based distribution.

The angular scattered light intensity largely depends on the optical properties of the particles. For small particles whose radiuses lower than $10 \mu \mathrm{m}$, the refractive index 
dependence becomes significant because at such small sizes the light irradiated onto the particle is not completely absorbed and can emerge as a refracted ray. In this case, the Mie theory should be used instead of the Fraunhofer theory, which does not take into account the optical properties of the particles. When examining the activated sludge floc size, the optical poly-disperse properties are difficult to be characterized and the Fraunhofer theory has to be used. The section from Mie to Fraunhofer needs to be revised by other method, beyond all question, the image technique is good approach.

The techniques based on laser light scattering are more suited to follow the fast changes that may occur in floc size during the process. Since the light scattering method doesn't usually offer visual information, coupling it to an image analysis system allows a direct visual inspection of the process evolution. If combine light scattering and CCD video microscope, not only the classification of particle shape can be realized, also the comparison and analysis of results between experiment and calculation by corresponding shape can bring more information which impossible received by individual method. The chapter describes a new instrument, aerosol particle shape and scattering analyzer based on imaging. By analyzing scattering intensity coefficient and polarization of fibre cotton and calculation from wave theory, the affecting factors are pointed out.

\section{Description of the instrument}

Figure 3 shows the experimental apparatus to measure the shape and scattering properties of aerosol particles in analog manner at the semiconductor laser wavelength of $0.65 \mathrm{um}$. The instrument realizes the combination of imaging and light scattering ${ }^{[19]}$. The scattering chamber, a homocentric hollow black sphere showed in Fig.3, is the core portion of the analyzer based on imaging. The hollow black sphere is composed by two symmetrical hollow hemispheres, which fabricated of aluminium considering hardness and weight. The interior wall of the hollow sphere is made coarse elaborately to reduce the influence from its scattering and reflection. The chamber inner diameter is $48 \mathrm{~mm}$, and the outer diameter is $76 \mathrm{~mm}$. There is a large aperture at top and bottom on the vertical radial line of the chamber, aerosol particle inlet and aerosol particle outlet respectively. The apertures at front and back on the horizontal radial line are respectively for assembling semiconductor laser and CCD video microscope. Without saturation of CCD, there is a filter corresponding laser wavelength in front of microscope. 36 small apertures for optical fibre that are positioned to measure the light from the horizontal and vertical scattering angles between $30^{\circ}$ and $150^{\circ}$ in $15^{\circ}$ increments. The diameter of each aperture is $3.02 \mathrm{~mm}$, which is slightly greater than diameter of optical fibre. The laser plane of polarization is set perpendicular to the horizontal plane. The inner diameter of the aperture port near the interior wall of scattering chamber, whose thickness is $1 \mathrm{~mm}$, is a little smaller than outer diameter of optical fibre, and the all ports for collecting scattering light can be strictly same distance from the centre of the chamber. The axeses of all apertures are directing the centre of the scattering chamber. The respective horizontal and vertical 18 small apertures are symmetrical about horizontal radial line. Each optical fibre collects the scattering light with an acceptance angle of $\pm 1.5^{\circ}$ since 
assembled a convex lens with $2.5 \mathrm{~mm}$ diameter. Unfortunately, the small apertures on the vertical radial line happen to be concurrent with sample pipe and waste pipe.

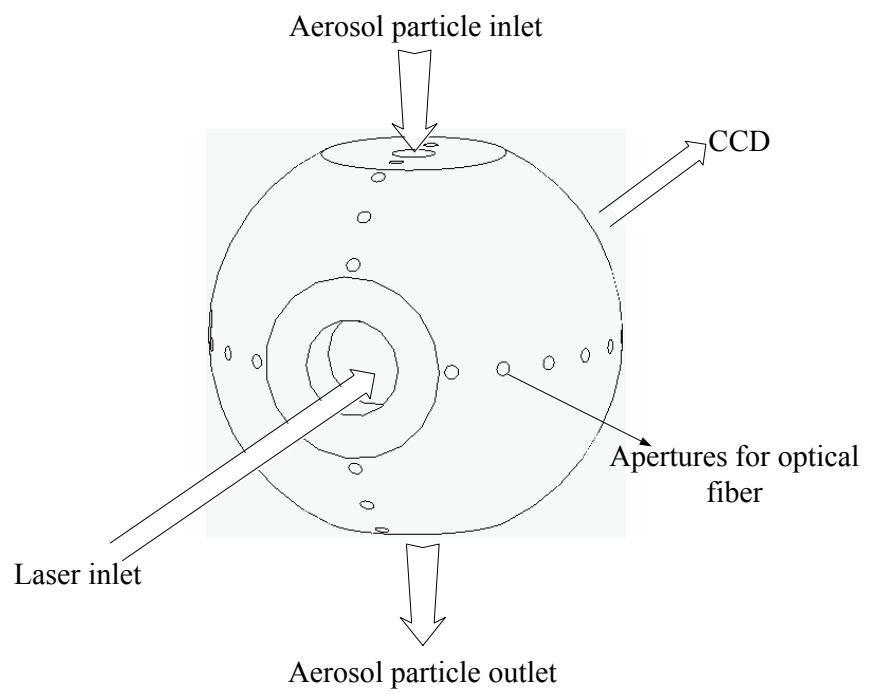

Figure 3. Simplified schematic map of aerosol particle shape and scattering analyzer

The scattering chamber must be puffed by clean air to eliminate the influence from the impurity. Single aerosol particle stream vertical to the laser beam is drawn in through the scattering chamber along the axes of aerosol particle inlet and outlet, and is ensheathed by filtered air drawn in through ports simultaneously. A set of filters and regulators introduce aerosol particles entrained in a fine laminar stream through the center of the chamber and intersecting the laser beam one particle at a time. Individual particles in the stream produce pulses of scattered light, which are amplified by photomultiplier tube detectors connected to a corresponding optical fibre bundles.

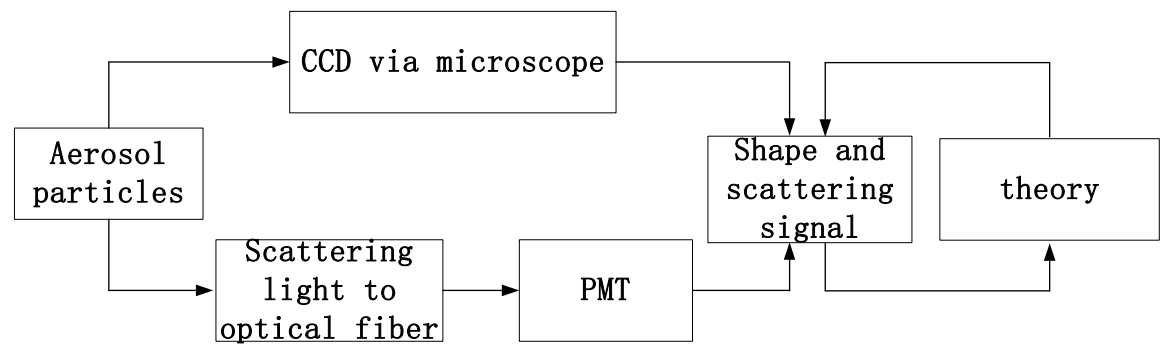

Figure 4. Working process of aerosol particle shape and scattering analyzer

The whole working process is described as Fig 4 . The integration time of CCD is slightly less than $0.005 \mathrm{~s}$. During experiment, the laser beam diameter is $1.5 \mathrm{~mm}$, and wavelength is $650 \mathrm{~nm}$. When single particle stream passing chamber center, the image is immediately 
acquired by the CCD video microscope, at the same time, the scattering light of corresponding particle is collected by optical fibers and transmitted to PMT. The speeds of aerosol particles are controlled by pressure difference of inner and outer hollow sphere. By adjusting the pressure difference, the particle speeds can be restrained less than $0.4 \mathrm{~m} / \mathrm{s}$ for effective diameter higher than $1 \mu \mathrm{m}$.

Since scattering light contains the information about shape of particle, more significant conclusion can be obtained by comparing experimental results and calculation from theory. The shapes of aerosol particles can be deduced through scattering light distribution, and the result will be Verified by corresponding images from CCD. So the data library of scattering and image about aerosol particles is gradually built, moreover, the aerosol particles are classified according to relevant shape and size.

\section{Result and discussion}

When the aspect radio exceeds 10, fiber particle, a common shape sort in aerosol, can be considered infinitely long cylinder. Generally, particles with effective radius less than 10um are inhaled ${ }^{[20]}$. However, in 1990, the U.S. National Institute for Occupational Safety and Health stated "no evidence for a threshold or 'save' level of asbestos exposure ${ }^{[21]}$. Flying particles are inclined to keep their long axes consistent with axes of carried gas, which ensures scattering light relatively steady.

\subsection{Wave theory for infinitely long cylinder}

The scattering geometry of infinitely long cylinder is shown in Fig.5. The $z$ axis of the cylindrical coordinates $(r, \phi, z)$ is defined along the central axis of the cylinder. The angle between the incident ray and the negative $z$ axis is denoted as $\chi$. $\alpha$ is defined as an oblique incident angle which is the complement angle of $\chi$. The $x$ axis is defined in the plane containing the direction of the incident ray and the $z$ axis. This plane defines the angles $\phi=0$ and $\phi=\pi$. The coordinate $r$ is then contained on the $x y$ plane such that the cylinder occupies the region $r \leq a$, where $a$ is the cross-section radius of the cylinder ${ }^{[22,23]}$. To illustrate the scattering geometry, a cylinder whose diameter is larger than the incident wavelength $\lambda$ so that the geometric optics will be used. The rays externally reflected, refracted, and internally reflected on the surface of the cylinder follow the Snell laws.

The scattering angle $\theta$, which is defined as the angle between the direction of the incident wave and the scattered wave, is obtained:

$$
\cos \theta=\sin ^{2} \alpha+\cos ^{2} \alpha \cos \phi
$$

$\phi$ is defined as an observation angle to distinguish it from the scattering angle. $\phi$ and $\theta$ are equal only at normal incidence. In all other cases, the values of $\phi$ are always more than that of $\theta$. So there is no true backscattering for an infinitely long cylinder. Starting from 
Maxwell equations, after complex algebraic operations, the scattering coefficients $a_{n}$ and $b_{n}$ are deduced as below:

$$
\begin{aligned}
& b_{n 1}=P_{n} \frac{Q_{n}^{2}+A_{n}\left(\varepsilon_{1}\right) B_{n}\left(\varepsilon_{2}\right)}{Q_{n}^{2}+A_{n}\left(\varepsilon_{1}\right) A_{n}\left(\varepsilon_{2}\right)} \\
& a_{n 2}=P_{n} \frac{Q_{n}^{2}+B_{n}\left(\varepsilon_{1}\right) A_{n}\left(\varepsilon_{2}\right)}{Q_{n}^{2}+A_{n}\left(\varepsilon_{1}\right) A_{n}\left(\varepsilon_{2}\right)} \\
& a_{n 1}=-b_{n 2}=P_{n} Q_{n} \frac{A_{n}\left(\varepsilon_{1}\right)-B_{n}\left(\varepsilon_{1}\right)}{Q_{n}^{2}+A_{n}\left(\varepsilon_{1}\right) A_{n}\left(\varepsilon_{2}\right)}
\end{aligned}
$$

Where

$$
\begin{aligned}
& A_{n}\left(\varepsilon_{1,2}\right)=j \frac{H_{n}^{(2) '}(l a)}{H_{n}^{(2)}(l a)}-\varepsilon_{1,2} l \frac{J_{n}^{\prime}(j a)}{J_{n}(j a)} \\
& B_{n}\left(\varepsilon_{1,2}\right)=j \frac{J_{n}^{(2)^{\prime}}(l a)}{J_{n}^{(2)}(l a)}-\varepsilon_{1,2} l \frac{J_{n}^{\prime}(j a)}{J_{n}(j a)},\left\{\begin{array}{c}
\varepsilon_{1}=1 \\
\varepsilon_{2}=m^{2}
\end{array}\right. \\
& P_{n}=J_{n}(l a) / H_{n}^{(2)}(l a) \\
& Q_{n}=i n h\left(l^{2}-j^{2}\right) / x l j \\
& x=k a=2 \pi a / \lambda
\end{aligned}
$$

If $\alpha=0^{\circ}$, then $a_{n 1}=b_{n 2}=0$. It should be noted that these coefficients depend on the refractive index, the size parameter and the oblique incident angle.

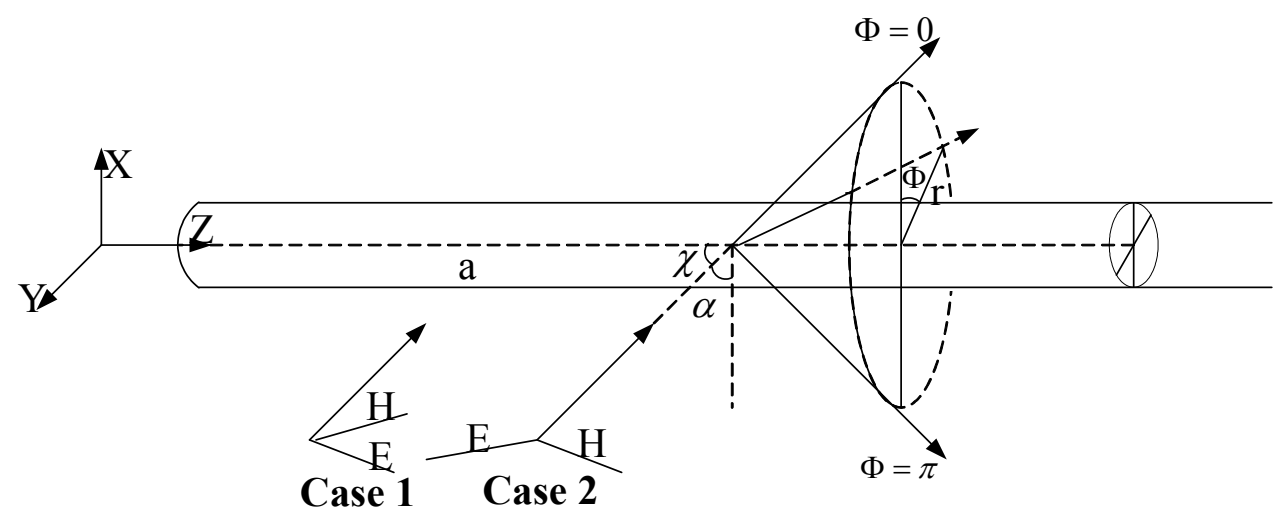

Figure 5. Geometry for light scattered by an infinitely long cylinder

Now we shall consider two simple cases separately. First, the electric vector $\vec{E}$ is parallel to the incident plane. This is sometimes called the TM mode. For the second case, the electric vector $\vec{E}$ is perpendicular to the incident plane and called the TE mode. The intensities of the scattered light in any direction are: 


$$
\begin{gathered}
I_{T M-11}=2 i_{11} I_{0} / \pi k R \\
I_{T M-12}=2 i_{12} I_{0} / \pi k R \\
I_{T E-22}=2 i_{22} I_{0} / \pi k R \\
I_{T E-21}=2 i_{21} I_{0} / \pi k R
\end{gathered}
$$

The intensity coefficients for above two cases are defined as:

$$
\mathbf{T M}\left\{\begin{array} { l } 
{ i _ { 1 1 } = | b _ { 0 1 } + 2 \sum _ { n = 1 } ^ { \infty } b _ { n 1 } \operatorname { c o s } n \phi | ^ { 2 } } \\
{ i _ { 1 2 } = | 2 \sum _ { n = 1 } ^ { \infty } a _ { n 1 } \operatorname { s i n } n \phi | ^ { 2 } }
\end{array} \quad \mathbf { T E } \left\{\begin{array}{l}
i_{22}=\left|a_{02}+2 \sum_{n=1}^{\infty} a_{n 2} \cos n \phi\right|^{2} \\
i_{21}=\left|2 \sum_{n=1}^{\infty} b_{n 2} \sin n \phi\right|^{2}
\end{array}\right.\right.
$$

where $a_{n 1}, b_{n 2}, a_{02}$ and $b_{01}$ are scattering coefficients. $i_{11}$ and $i_{22}$ are the scattered intensities that lies in the same plane as the incident intensities, while $i_{12}$ and $i_{21}$ are the cross-polarized scattered intensities that have directions perpendicular to the incident intensities, what's more, $i_{12}=i_{21}$.

The polarization of scattering light is defined as ${ }^{[24]}$ :

$$
\mathrm{TM}: p_{11}=\frac{i_{11}-i_{12}}{i_{11}+i_{12}} \quad \text { TE: } p_{22}=\frac{i_{22}-i_{21}}{i_{22}+i_{21}}
$$

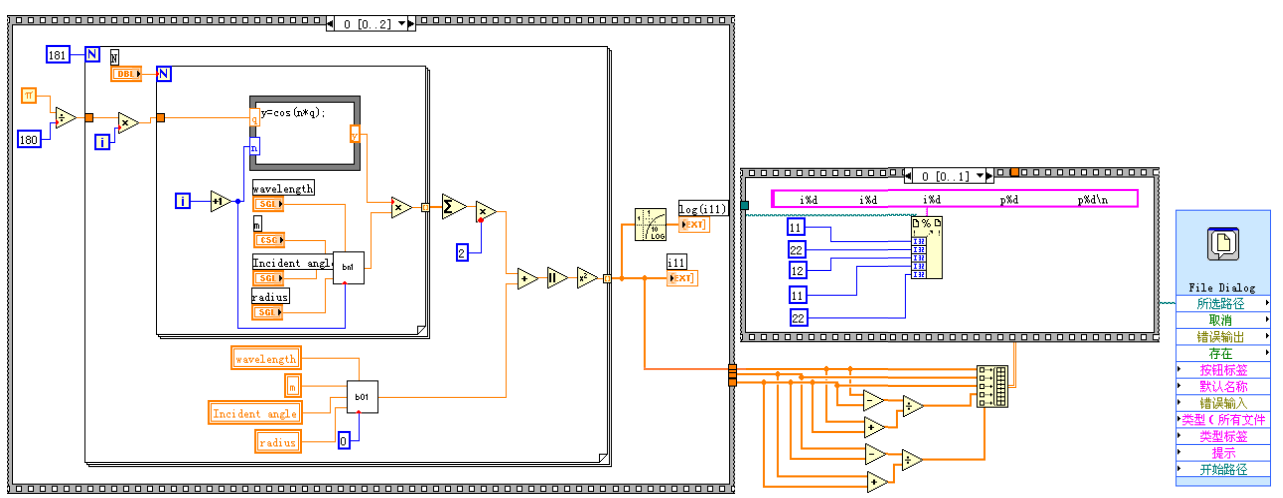

Figure 6. Schematic of programming wave theory

LabVIEW is a graphical programming language which has its roots in data acquisition and automation control. Its graphical representation, similar to a process flow diagram was created to provide an intuitive programming environment for users ${ }^{[25]}$. The language has matured over the last twenty years to become a general purpose programming environment. With LabVIEW, we have self-programmed wave theory as a part of the whole measuring system. The programming structure is demonstrated partly in Fig.6. The program consists of 3 relevant parts, which are similar at format. The calculation data, which is function of incident angle, 
refractive index, incident wavelength and cylinder diameter, is saved in form of .txt. The data will be more analysed through Origin software and compared with information from structure according to shape given by CCD via microscope.

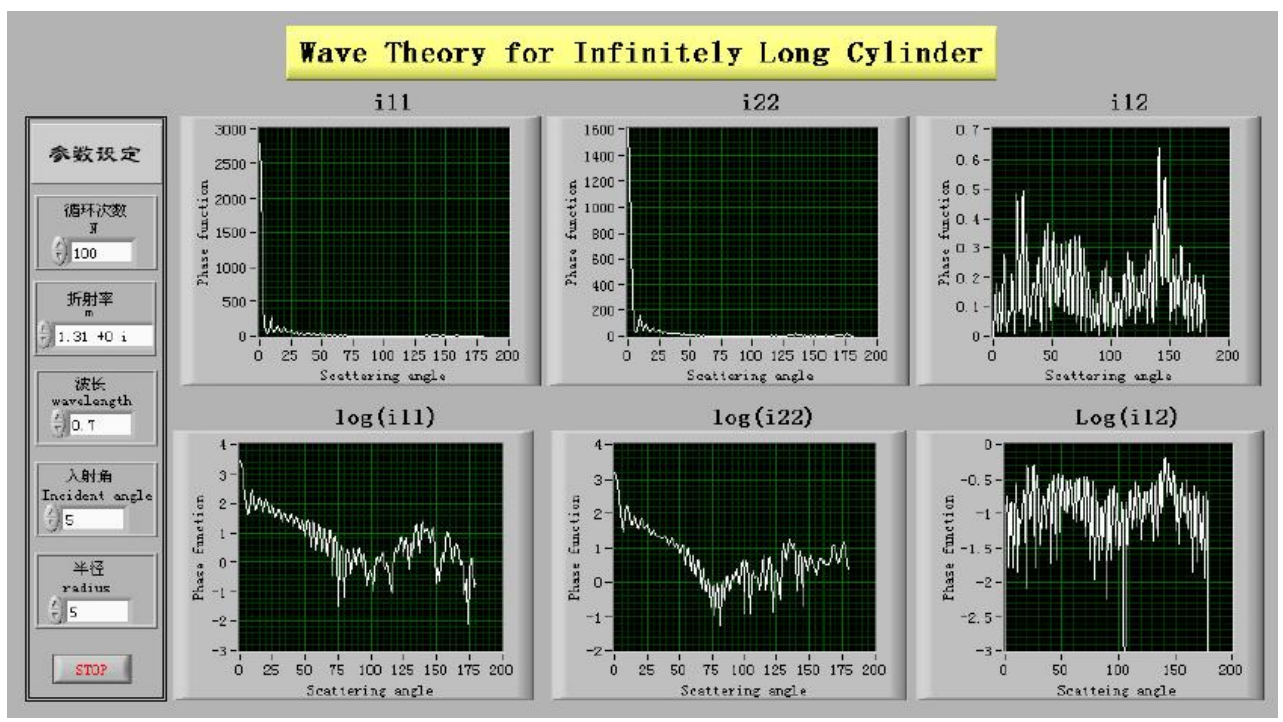

Figure 7. Interface of programming wave theory

The interface of the program for calculation is shown in Fig.7, which contains input parameter area and display windows of calculating results. The display windows contain $i_{11}$, $i_{22}$ and $i_{12}$, also their logarithmic format. The input parameters include refractive index, laser wavelength, incident angle and radius of cylinder. In a general way, the whole process for calculation is shorter than 30s. With the increase of incident angle, the calculation time will be extended slightly.

\subsection{Analysis of experiment and calculation}

The effective radius of selected fiber cotton particles for experiment are about 10um, compared to $1 \mathrm{~mm}$ laser beam, the condition of infinitely long for irradiated cotton is satisfied. The incident laser with $0.65 \mathrm{um}$ wavelength is transformed to linear polarized light by Glan-Talyor lens. When electric vector $\vec{E}$ is parallel to the incident plane, the refractive index of fibre cotton is 1.573-1.581, we choose the middle number 1.577 for calculation. When the electric vector $\vec{E}$ perpendicular to the incident plane, the corresponding refractive index is 1.524-1.534, we also choose the middle number 1.529 for calculation. In experiment, the angle between cotton and axes of carried gas is about $5^{\circ}$.

The "left, right, up and down" in figure 4 refer to figure 1.It can be concluded that the tendency of experiment data keeps uniform with calculation of the infinitely long cylinder for scattering intensity and polarization. A pair of experimental signal lack in polarization 
$\mathrm{P}_{11}$ and $\mathrm{P}_{22}$, since up aperture and aerosol particle inlet with a same positions, similarly to bottom aperture and aerosol particle outlet, too. $\mathrm{i}_{11}$ and P11are more close to calculation than $i_{22}$ and $P_{22}$, which might be caused by different outline of fibre cotton particles. Clearly, the difference between cotton fibre in image A and infinitely long cylinder is smaller than that between image B and infinitely long cylinder. The experiment is cursory in describing trendy of scattering intensity and polarization restricted by the number of optical fibre for collecting scattering light; on the one hand, the angle increments between apertures for optical fibre are limited in manufacturing process; on the other hand, the cone angle of receiving plane for every optical fibre is $3^{\circ}$, unlike the elements of calculation, the integral photometric characteristics are much less dependent on particle shape.

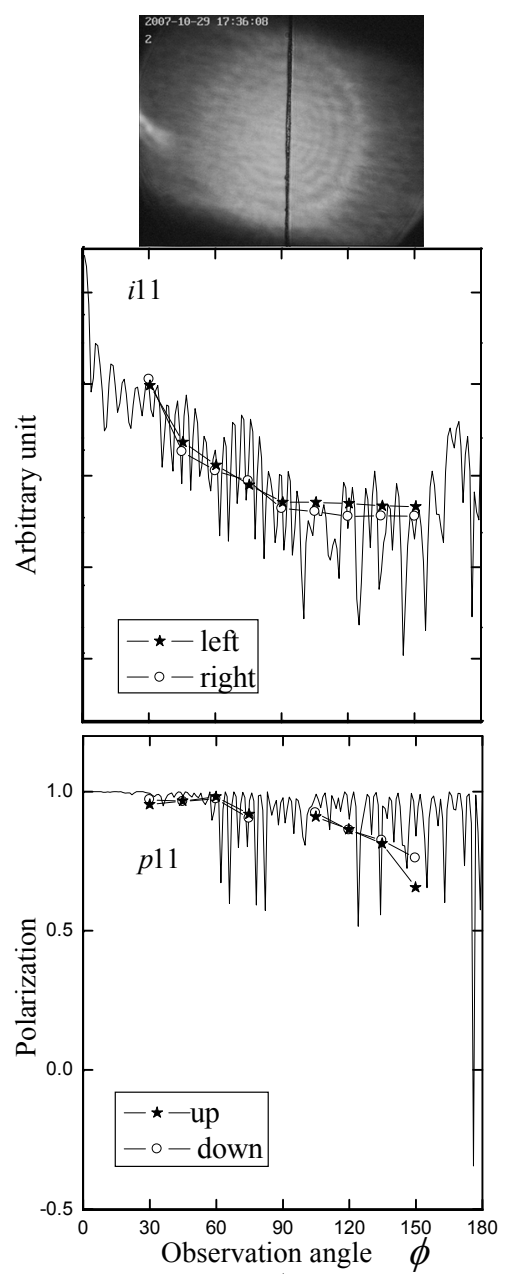

A

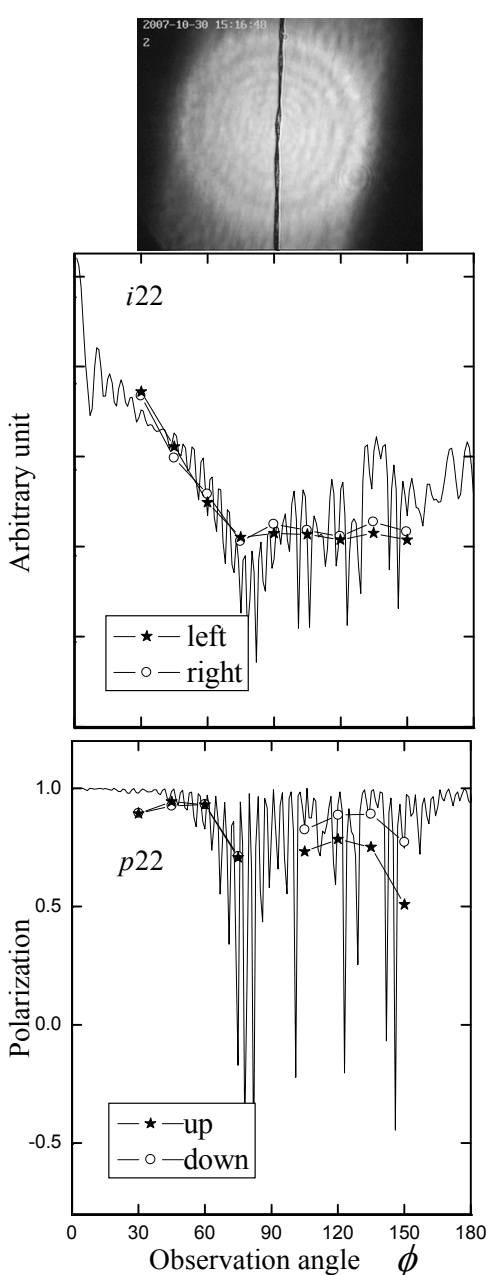

B

Figure 8. Photo of fiber micro-particle by $\mathrm{CCD}$ and respective scattering and polarization 


\section{Conclusion}

An experimental apparatus has been built to measure the images and light scattering characteristics of aerosol particles simultaneously. The core portion of the analyzer is a homocentric hollow black chamber. Images, corresponding scattering intensity and polarization of fiber cottons are received. Wave theory for infinitely long cylinder has been compiled with LabVIEW. By comparison of experimental data and calculation, the affecting factors to results are pointed out, which provides a good foundation to further study.

\section{Author details}

Shiyong Shao*, Yinbo Huang and Ruizhong Rao

Key Laboratory of Atmospheric Composition and Optical Radiation, Anhui Institute of Optics and Fine Mechanics, Chinese Academy of Sciences, China

\section{Acknowledgement}

The authors are very thankful to the reviewers for valuable comments. This work was supported by Youth Talent fund from Hefei Institutes of Physics Science under Contract No. Y03AG31141. The theoretical calculations in this paper have been kindly assisted by Lei Hao and Yongbang Yao helped with the construction of the apparatus.

\section{References}

[1] Shao, S.Y., Huang, Y.b., Yao, Y.b., Rao, R.Z., "Progress in Shape Measurement Technology of Micro-particles in Atmosphere, " Journal of Atmospheric and Environmental optics 3(1), 1-10(2008).

[2] Mishchenko, M.I., Lacis, A.A., "Scattering, absorption, and emission of light by small particles, " Cambridge university press, New York, 279-359 (2002).

[3] Zhao, J.qi, Shi, G.Y.., "Light Scattering Properties of Snall Nonspherical Particles, " Science Technology and Engineering., 5(24):1872-1875 (2005).

[4] Edwin, H., Paul, H.K., John, R.G.., "Light scattering from non-spherical airborne particles: experimental and theoretical comparisons, " Appl. Opti., 33(3), 71807186(1994).

[5] Mi, F.W., "The laser diffraction particle size analyzer for methods to calculate particle size distribution," Acta Photonica Sinica, 28(2), 151-154(1999).

[6] Li, L.F., Zhang, L., Dong, L., "Experimental Study of the Concent ration of Soot Based on the Method of Optical Back Scattering, " Acta Photonica Sinica, 35 (6), 9152918(2006).

[7] Liu, J., Hua, D.X., L I, Y., “Ultraviolet lidar for profiling of $\mathrm{t}$ he urban atmospheric aerosol spatial and temporal at Xi'an, " Acta Photonica Sinica, 36 (8), 153421537(2007).

[8] Diehl, S.R., Smith, D.T., Sydor, M., “Analysis of suspended solids by single-particle scattering, " Appl.Opt, 18(10), 1653-1658 (1979).

${ }^{*}$ Corresponding Author 
[9] Bartholdi, M., Salzman, G.C., Hiebert, R.D., Kerker, M., “Differential light scattering photometer for rapid analysis of single particles in flow, " Appl.Opt, 19(10), 15731581(1980).

[10] Kaye, P.H., Eyles, N.A., Ludlow, I.K., “An instrument for the classification of airborne particles on the basis of size, shape and count frequency, " Atmospheric environment, 25(3), 645-654(1991).

[11] Kaye, P.H., "Spatial light-scattering analysis as a mean of characterizing and classifying non-spherical particles, " Meas.Sci.Technol, 9, 141-149(1998).

[12] Foot, V.E., Clark, J.M., Baxter, K.L., Close, N., “Characterising single airborne particles by fluorescence emission and spatial analysis of elastic scattered light, " in Optically Based Biological and Chemical Sensing for Defence. Proc.SPIE., 5617, 222-299(2004).

[13] Barbusinski, K., Koscielniak, H., "Influence of substrate loading intensity on floc size in the activated sludge process, " Wat.Res., 29(7), 1703-1710(1995).

[14] Grijspeerdt, K., Verstraete, W., "Image analysis to estimate the settleability and concentration of activated sludge," Wat.Res., 31(6), 1126-1134(1997).

[15] Arjen Van Der Schoot, "Dual-channel particle size and shape analyzer, "China Particuology, 2(1), 44-45 (2004).

[16] Govoreanu, R, Vandegehuchte, K, Saveyn, H, “An automated image analysis system for on-lines structural characterization of the activated sludge flocs, "Med. Fac. Landbouww. Univ. Gent" 67(4), 175-178(2002).

[17] Govoreanu, R., Saveyn, H., Meeren, P.V.D, Vanrolleghem, P.A., “Simultaneous determination of activated sludge floc size distribution by different techniques, "Water Sci. Technol., 50(12), 39-46(2004).

[18] Allen T., "Particle size measurements, "Fifth edition. Chapman and Hall Ltd., London.

[19] Shao, S.Y., Yao, Y.b., Rao, R.Z., "New instrument for detecting shape and scattering of micro-particles based on imaging," China Patent, No:200710023960.X.

[20] Liu, J.J., Zhang, W.G., “Design and Investigation of Method for Accuracy Determination of Inhalable Particulate Matter Sampler, "China powder science and technology, 5(12):5-8(2006).

[21] Paul, K., Edwin, H., Zhenni, W.T., "Neural-network-based spatial light-scattering instrument for hazardous airborne fiber detection, " Appl. opt, 36(24), 6149-6156(1997).

[22] Liou, K.N., “Electromagnetic scattering by arbitrarily oriented ice cylinders, " Appl. Opti., 11(3), 667-674 (1972).

[23] Van de Hulst H.C., "Light scattering by small particles, " John Willey \& Sons, New York, 297-328(1957).

[24] Zhao, K.H., Zhong, X.H., “Optics”(Volume one). Beijing: Beijing University Press, 235245(2004).

[25] Zhou, Y.H., Wang, Y.N., Wu, W.C., "Design and implementation of voltage-current testing system based on LabVIEW for photovoltatic cells, " Chinese Journal of Scientific Instrument, 27(6), 1775-1776(2006). 